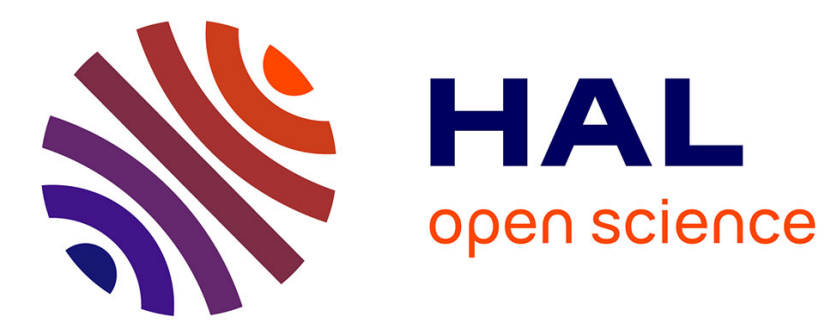

\title{
Ponderomotive Forces Near the Dayside Magnetospheric Boundary
}

\author{
O. Pokhotelov, F. Feygin, L. Stenflo, P. Shukla
}

\section{To cite this version:}

O. Pokhotelov, F. Feygin, L. Stenflo, P. Shukla. Ponderomotive Forces Near the Dayside Magnetospheric Boundary. Journal de Physique IV Proceedings, 1995, 05 (C6), pp.C6-49-C6-52. 10.1051/jp4:1995609 . jpa-00253972

\section{HAL Id: jpa-00253972 https://hal.science/jpa-00253972}

Submitted on 1 Jan 1995

HAL is a multi-disciplinary open access archive for the deposit and dissemination of scientific research documents, whether they are published or not. The documents may come from teaching and research institutions in France or abroad, or from public or private research centers.
L'archive ouverte pluridisciplinaire HAL, est destinée au dépôt et à la diffusion de documents scientifiques de niveau recherche, publiés ou non, émanant des établissements d'enseignement et de recherche français ou étrangers, des laboratoires publics ou privés. 


\title{
Ponderomotive Forces Near the Dayside Magnetospheric Boundary
}

\author{
O.A. Pokhotelov, F.Z. Feygin, L. Stenflo* and P.K. Shukla** \\ Institute of Physics of the Earth, Russian Academy of Sciences, 123810 Moscow, Russia \\ * Department of Plasma Physics, Umeå University, 90187 Umeå Sweden \\ ** Institut für Theoretische Physik IV, Ruhr-Universität Bochum, 44780 Bochum, Germany
}

\begin{abstract}
It is shown that the existence of the so-called "magnetic holes" (magnetic field minima) along the field lines play a significant role in the plasma pressure balance in the Earth's magnetosphere. Ponderomotive forces induced by the Pcl geomagnetic pulsations propagating through these regions can lead to a significant plasma accumulation there.
\end{abstract}

\section{INTRODUCTION}

Investigations of ponderomotive forces induced by geomagnetic pulsations have shown that they can significantly contribute to the plasma balance in the Earth's magnetosphere [1-4]. It is known [2-4] that if the amplitude of the $\mathrm{Pcl}$ pulsations exceeds some critical value then a pronounced maximum of the plasma density is formed in the vicinity of the apex of the Pc1 trajectory. It is evident that its position coincides with a minimum of the dipole magnetic field along that trajectory.

In previous studies this effect was analyzed with the dipole approximation in the close vicinity of the equatorial plane and near high latitudes where the geomagnetic field lines are nearly radial. However, such approximations are justified only at small altitudes. The magnetic data collected by satellites, and theoretical models of the geomagnetic field show a complex structure of the geomagnetic field at the midday boundary of the Earth's magnetosphere. This structure is characterized by a smooth transition from a " $V$ " to a " $W$ " magnetic field shape with two minima in the magnetic field along the field lines [5] This brings up the question whether the existence of two maxima in the distribution of the geomagnetic field along the field lines leads to the appearance of two maxima in the distribution of the plasma density along the Pel trace, and, consequently, how this will influence the conditions for $\mathrm{Pc1}$ generation and propagation. In this paper, we shall consider the ponderomotive force action induced by the geomagnetic pulsations in the dayside magnetosphere.

\section{GOVERNING EQUATIONS}

In a spherical coordinate system, the magnetic field components for the two-dipole model in the meridional noon-midnight plane have the form

$$
B_{r}=-\frac{2 B_{E} x}{r^{3}} \alpha,
$$

and

$$
B_{\varphi}=\frac{B_{E}}{r^{3}} \sqrt{1-x^{2}} \beta
$$


where $r$ is the geocentric radial distance ( $r$ is measured in units of the Earth's radius $R_{E}$ ), $x=\sin \varphi$, $\varphi$ is the geomagnetic latitude, $B_{E}$ is a constant ( $B_{E}=0.3$ gauss), and

$$
\alpha=1-\frac{k r^{3}\left(a^{2}-2 r^{2}+a r \sqrt{1-x^{2}}\right)}{2\left(a^{2}+r^{2}-2 a r \sqrt{1-x^{2}}\right)^{5 / 2}},
$$

and

$$
\beta=1+\frac{k r^{3}\left(\sqrt{1-x^{2}}\left(a^{2}+r^{2}\right)-a r\left(2+x^{2}\right)\right)}{\sqrt{1-x^{2}}\left(a^{2}+r^{2}-2 a r \sqrt{1-x^{2}}\right)^{5 / 2}},
$$

where $k$ and $a$ are two constant model parameters [5]. Thus, the magnetic field at an arbitrary point at the field lines and the equation for the field line are determined by

$$
B=\frac{B_{E}}{r^{3}}\left[4 x^{2} \alpha^{2}+\left(1-x^{2}\right) \beta^{2}\right]^{1 / 2}
$$

and

$$
d r / d x=-\frac{2 r x}{\left(1-x^{2}\right)} \frac{\alpha}{\beta}
$$

Here we consider the case where the axis of the original and the additional dipoles are parallel to each other. The analysis will then reveal the existence of two minima of the magnetic field which are shifted from the equator of the field line near the dayside boundary of the magnetosphere. This is in contrast to the one-dipole model with the minimum of the magnetic field at the equator. That picture is also confirmed by satellite observations. Below we choose the parameters $a=33$ and $k=13$, which corresponds to the dayside boundary of the magnetosphere at $10 R_{E}$. When $a$ is approaching infinity, we have a limiting transition to the one-dipole approximation.

Let us now consider the action of the ponderomotive force $f_{\|}$induced by the ion-cyclotron waves propagating along the magnetic field lines [6]. The longitudinal component of this force can be written in the form [2]

$$
f_{\|}=-\frac{n^{2} E^{2}}{16 \pi}\left[\frac{\omega}{\Omega_{i}-\omega} \nabla_{\|} \ln B+\nabla_{\|} \ln \rho\right],
$$

where

$$
n^{2}=\frac{\omega_{0 i}^{2}}{\Omega_{i}\left(\Omega_{i}-\omega\right)}+1 .
$$

Furthermore, $E$ is the wave electric field, $\rho$ is the plasma mass density, $\Omega_{i}$ is the ion cyclotron frequency, $n$ is the refractive index and $\omega_{0 i}$ is the ion plasma frequency. In the WKB approximation $E \sim(B / n)^{1 / 2}$, where $B$ and $n$ are evaluated at the axis of the field tube.

In order to determine the plasma distribution along the geomagnetic field line we consider the force balance in the longitudinal direction with respect to the geomagnetic field, or

$$
\nabla_{\|} P=\rho g_{\|}+f_{\|},
$$

where $P=\rho c_{s}^{2}$ is the plasma pressure and $c_{s}$ is the sound velocity. The plasma is supposed to be isothermal and to be composed of ions of one species (protons) and electrons. The operator $\nabla_{\|}$ can be replaced by $(d x / d l) d / d x$, where $d l$ is the element of the field line arc. It is determined by $d l=\left[r^{2} /\left(1-x^{2}\right)+(d r / d x)^{2}\right]^{1 / 2} d x$. Finally, the longitudinal component of the gravity force is

$$
g_{\|}=\frac{B_{r}}{B} \frac{g_{E}}{r^{2}}
$$

where $g_{E}=980 \mathrm{~cm} / \mathrm{s}^{2}$ and $B_{r}$ and $B$ are defined by the relations (1) and (5).

Substituting (7) and (9) into (8) we the find a differential equation of first order which determines the distribution of the plasma density along the field line. Thus

$$
\frac{d N}{d x}=\frac{A_{1} N^{3 / 2}+A_{2} N}{N^{1 / 2}+A_{3}}
$$


where

$$
\begin{gathered}
A_{1}=\frac{2 g_{E} R_{E} x}{c_{s}^{2}\left(1-x^{2}\right) r} \frac{\alpha}{\beta}, \\
A_{2}=-\frac{\nu_{0} A_{3}}{\left(1-\nu_{0} B_{0} / B\right)} \frac{B_{0}}{B^{2}} \frac{d B}{d x}
\end{gathered}
$$

and

$$
A_{3}=\frac{b_{0}^{2}\left(1-\nu_{0}\right)^{1 / 2}}{16 \pi \rho_{0} c_{s}^{2}\left(1-\nu_{0} B_{0} / B\right)^{1 / 2}} .
$$

Here $b_{0}$ is the oscillation amplitude of the magnetic field, $\nu_{0}=\omega / \Omega_{i 0}$ is the ratio of the wave frequency to the ion-cyclotron frequency in the apex of the field line, $B_{0}$ is the magnetic field at the equator and $N=\rho / \rho_{0}$, where $\rho_{0}$ is the plasma mass density at the equator.

\section{NUMERICAL RESULTS}

The integration of eq. (10) has been carried out by means of the Runge-Kutta method by assuming that the square of the sound velocity $c_{s}^{2}$ was $3 \times 10^{11} \mathrm{~cm}^{2} / \mathrm{s}^{2}$. The plasma mass density at the equator $\rho_{0}$ was chosen as $1.67 \times 10^{-23} \mathrm{~g} / \mathrm{cm}^{3}$. Furthermore, we take $\nu_{0}$ and $b_{0}$ to be constant $\left(\nu_{0}=0.7, b_{0}=3\right.$ $\mathrm{nT}$ ). The McIlwain parameter $L$ varies here from 6.1 to 9.5 . Our results for the plasma mass density redistribution show a smooth transition from a plasma condensation in the equatorial plane (the one-dipole approximation) to the appearance of plasma density maxima far from the equator along the field line in the vicinity of the field minima. Moreover, both the plasma density and the position of the maxima along the field line (with respect to the equator) increase when we move away from the Earth. This should be expected because the deviation of the geomagnetic field from the one-dipole structure is significant due to the influence of the surface currents on the magnetopause. Let us recall that the numerical example presented here corresponds to the case where the distance from the center of the Earth to the magnetopause is of the order of $10 R_{E}$. In this case the bifurcation, i.e., the transition from a $N(x)$ distribution with one minimum to a distribution with two maxima occurs at a distance of $8 R_{E}$. The variation of the solar wind pressure leads to a change of the magnetopause position and a subsequent change of the distance where bifurcation occurs.

The plasma density variations along the field line at $L=9.5$ and $b_{0}=3 \mathrm{nT}$ for different values of $\nu_{0}$ exhibit that the ponderomotive effect increases when $\nu_{0}$ increases. Finally, we observe that the increase of the pulsation amplitude leads to a significant amplification of the ponderomotive effect.

\section{DISCUSSION}

In this paper, we have performed analytical and numerical studies of the influence of the geomagnetic field structure near the dayside magnetospheric boundary on the plasma density redistribution due to the ponderomotive force action induced by the ion-cyclotron waves. We have demonstrated that the picture of plasma condensation may be significantly altered with a more general geometry for the magnetic field of the Earth. The transition from a " $V$ " to a " $W$ " structure of the Earth's magnetic field may lead to the appearence of so called "magnetic holes" playing a significant role in the plasma density redistribution in the magnetosphere. Based on the Antonova and Shabansky model [5] for the geomagnetic field we have thus found a new effect which results in the appearence of two plasma density maxima in the regions of the "magnetic holes". The existence of such regions can also lead to anomalous resonant absorption of the ion-cyclotron waves (the "magnetic beach" effect), which may be the reason for the partial (or even complete) absence of ion-cyclotron wave spectra at high latitude observatories. 


\section{Acknowledgments}

This work was partially supported by the Commission of the European Union (Brussels) through Research Grant No. INTAS-94-870, and through the network "Microphysics of Nonlinear Phenomena in Collisionless Plasmas: Application to Space and Laboratory Plasmas" of the Human Capital and Mobility Program under Contract no. CHRX-CT-93-0356. The authors are grateful to Prof. A. V. Guglielmi, Dr. E. N. Fedorov, and Dr. G.Brodin for useful discussions.

\section{References}

[1] Allan W., J. Geophys. Res. 97 (1992) 8483.

[2] Guglielmi A. V., Pokhotelov O. A., Stenflo L., and Shukla P. K., Astrophys. Space Sci. 200 (1993) 91.

[3] Guglielmi A. and Pokhotelov O. A., Space Sci. Rev. 65 (1994) 5.

[4] Guglielmi A. V., Pokhotelov O. A., Feygin F. Z., Kurchashov Yu P., McKenzie J. F., Shukla P. K., Stenflo L and Patapov A. S., J. Geophys. Res. 100 (1995) 7997.

[5] Antonova A. E. and Shabansky V. P., Geomagn. Aeron. 8 (1968) 639.

[6] Shukla P. K. and Stenflo L., Phys. Fluids 28 (1985) 1576. 